PROCEEDINGS OF THE

AMERICAN MATHEMATICAL SOCIETY

Volume 126, Number 11, November 1998, Pages 3239-3241

S 0002-9939(98)04385-8

\title{
THE CONVERGENCE OF THE FOURIER INTEGRAL OF A UNIMODAL DISTRIBUTION
}

\author{
CONSTANTINE GEORGAKIS
}

(Communicated by J. Marshall Ash)

\begin{abstract}
We show that the characteristic function of a unimodal probability distribution function can be inverted by the Fourier transform a.e. if and only if the distribution is absolutely continuous. The result complements Khintchine's criterion for unimodal distributions.
\end{abstract}

The distribution function $F(x)=\operatorname{Pr}(X \leq x)$ of a random variable $X$ is unimodal with vertex at the origin if $F(x)$ is convex for $x<0$ and concave for $x>0$. Some examples of unimodal distributions are: i) the standard normal, exponential and Cauchy probability densities; ii) the class of stable distributions; iii) the class of symmetric infinitely divisible distributions. According to Khintchine's criterion ([6]), $F$ is unimodal if and only if $X=U Z$ where $U, Z$ are independent random variables and $U$ is uniform on $[0,1]$, or equivalently, the characteristic function $\varphi(t)$ of $F$ is the integral average over $[0, t]$ of a characteristic function $\psi(t)$. The purpose of this note is to prove the following inversion Theorem for the characteristic function of a unimodal distribution. Medgyessy [8], Dharmadhikari and Kumar [2], and Bertin, Cuculescu and Theodorescu [1] provide an extensive survey on univariate and multivariate unimodal distributions.

Theorem. (a) The Fourier integral of an absolutely continuous unimodal distribution $F$ for $x \neq 0$ is convergent to $\left(F^{\prime}(x+0)+F^{\prime}(x-0)\right) / 2$, and to $F^{\prime}(x)$ except for a countable number of points.

(b) If the Fourier integral of a unimodal distribution is convergent a.e., then it is absolutely continuous.

Proof. Let $\varphi$ be the characteristic function of a unimodal distribution function $F$ on the real line, and define

$$
K(x, t)= \begin{cases}t^{-1} & \text { for } 0<x \leq t<\infty \\ -t^{-1} & \text { for }-\infty<t \leq x<0 \\ 0 & \text { otherwise }\end{cases}
$$

Received by the editors December 14, 1994 and, in revised form, March 10, 1997.

1991 Mathematics Subject Classification. Primary 42A38; Secondary 60E10.

Key words and phrases. Khintchine, unimodal distribution, Fourier integral, inversion, characteristic function.

(C)1998 American Mathematical Society 
By Khintchine's criterion ([7, pp. 92-95]), there exists a distribution function $G$, with characteristic function $\psi$, such that

$$
\varphi(x)=\int_{-\infty}^{\infty} e^{i x y} d F(y)=\frac{1}{x} \int_{0}^{x} \psi(t) d t=F(0)-F(0-)+\int_{-\infty}^{\infty} e^{i x y} f(y) d y
$$

where

$$
f(y)=\int_{-\infty}^{\infty} K(y, s) d G(s)
$$

for $-\infty<x, y<\infty$. It follows that $f \geq 0$ from (2). It also follows that

$$
\|f\|_{1}=1-G(0)+G(0-)=1-F(0)+F(0-)
$$

using (2) and Fubini's Theorem together with (1) when $x=0$. Moreover, $f$ is increasing on $(-\infty, 0)$, decreasing on $(0, \infty)$ by $(2)$, and $F$ is continuous except for the countable set of the discontinuity points of $G$, because from (2) we have the following inequalities:

$$
\begin{aligned}
& f(x)-f(x+t) \leq \frac{1}{x}(G(x+t)-G(x-0)), \quad x>0, t>0, \\
& f(x-t)-f(x) \leq \frac{1}{x-t}(G(x)-G(x-t-0)), \quad x>0,0<t<x, \\
& f(x+t)-f(x) \leq \frac{1}{-x-t}(G(x+t)-G(x-0)), \quad x<0,0<t<-x, \\
& f(x)-f(x-t) \leq \frac{1}{-x}(G(x)-G(x-t-0)), \quad x<0, t>0 .
\end{aligned}
$$

Thus, $f$ is Lebesgue integrable on $(-\infty, \infty)$ and of bounded variation on each finite interval in the complement of the origin. By the Dirichlet-Jordan inversion Theorem $([4$, p. 12]), it follows that the Fourier integral of $f$ is convergent for $x \neq 0$. That is,

$$
\lim _{r \rightarrow \infty} \frac{1}{2 \pi} \int_{-r}^{r} e^{-i x t} d t \int_{-\infty}^{\infty} e^{i t y} f(y) d y=\frac{f(x+)+f(x-)}{2}
$$

for $x \neq 0$, where $(f(x+)+f(x-)) / 2=f(x)$ except for the countable number of the discontinuity points of $G$. and

(a) Suppose $F$ is absolutely continuous. Then $F(0)-F(0-)=0$ in (1), $\|f\|_{1}=1$,

$$
\varphi(t)=\int_{-\infty}^{\infty} e^{i t y} f(y) d y, \quad-\infty<t<\infty .
$$

This makes $F$ the distribution function of the probability density $f$ by the uniqueness property of characteristic functions and (4). Therefore, the Fourier integral of $F$, which is identical with that of $f$ in (3), is convergent to $f(x)$ except for the countable number of the discontinuity points of $G$ and possibly the origin.

(b) Next, suppose the Fourier integral of $F$ is convergent a.e. Then it must be convergent to the derivative $F^{\prime}(x)$ a.e., since the Fourier integral of a distribution function is Cesaro summable to its derivative a.e. ([5, p. 153]). Thus,

$$
F^{\prime}(x)=\lim _{r \rightarrow \infty} \frac{1}{2 \pi} \int_{-r}^{r}\left(1-\frac{|t|}{r}\right) e^{-i x t} \varphi(t) d t=\lim _{r \rightarrow \infty} \frac{1}{2 \pi} \int_{-r}^{r} e^{-i x t} \varphi(t) d t
$$

a.e. for $-\infty<x<\infty$. But, the Fourier integral of $f$ is also convergent to $f(x)$ a.e. by (3). Hence, $F(0)-F(0-)=0$ in (1), and so (1) reduces to equation (4). Now, 
it follows as before that $F$ is the distribution function of the probability density $f$, and therefore absolutely continuous. This completes the proof.

Corollary. The characteristic function of an absolutely continuous unimodal distribution can be inverted via its Fourier transform to its probability density function everywhere except possibly for a countable number of points.

The Theorem is also valid for a unimodal distribution $F(x)$ with vertex at $a$, since $F(x+a)$ is a unimodal distribution with vertex the origin. The Corollary is also true for a distribution $F$, which is a countable mixture of absolutely continuous unimodal distributions $F_{j}$, that is,

$$
F(x)=\sum_{j=-\infty}^{\infty} p_{j} F_{j}(x), \quad p_{j} \geq 0, \quad \sum_{j=-\infty}^{\infty} p_{j}=1 .
$$

The author wishes to thank the referee for a helpful review of the first version of the paper.

\section{REFERENCES}

[1] E. M-J. Bertin, I. Cuculescu, and R. Theodorescu, Unimodality of Probability Measures, Kluwer Academic Publishers, 1997.

[2] S. Dharmadhikari and J-D. Kumar, Unimodality, Convexity, and Applications, Academic Press, 1988. MR 89k:60020

[3] C. Georgakis, The Hausdorff Mean of a Fourier-Stieltjes Transform, Proc. Amer. Math. Soc. 116 (1992), 465-471. MR 92m:42019

[4] R. R. Goldberg, Fourier Transforms, Cambridge University Press, 1962. MR 22:11254

[5] T. Kawata, Fourier Analysis in Probability Theory, Academic Press, 1972. MR 57:4284

[6] A. Ya. Khintchine, On Unimodal Distributions, Izv. Nauchno Issled. Inst. Mat. Mech. Temsk. Gos. Univ. 2, no. 2, 1-7 (1938).

[7] E. Lukacs, Characteristic Functions, Hafner Publishing Co., 1970. MR 49:11595

[8] P. Medgyessy, Decomposition of Superpositions of Density Functions and Discrete Distributions, John Wiley \& Sons, 1977. MR 55:13532b

Department of Mathematics, DePaul University, Chicago, Illinois 60614 